\title{
LETTERS
}

The purpose of this Letters section is to provide rapid dissemination of important new results in the fields regularly covered by Physics of Plasmas. Results of extended research should not be presented as a series of letters in place of comprehensive articles. Letters cannot exceed three printed pages in length, including space allowed for title, figures, tables, references and an abstract limited to about 100 words. There is a three-month time limit, from date of receipt to acceptance, for processing Letter manuscripts. Authors must also submit a brief statement justifying rapid publication in the Letters section.

\section{Transition to turbulence in a crossed-field gap}

\author{
P. J. Christenson and Y. Y. Lau \\ Intense Energy Beam Interaction Laboratory, Department of Nuclear Engineering, University of Michigan, \\ Ann Arbor, Michigan 48109-2104
}

(Received 5 July 1994; accepted 9 September 1994)

\begin{abstract}
The transition from laminar to turbulent behavior of the electron sheath in a cross-field gap is examined for the regime $B>B_{\mathrm{H}}$, where $B$ is the external magnetic field and $B_{\mathrm{H}}$ is the Hull cutoff value. An analytic expression is presented for the critical emitted current beyond which laminar solutions cease to exist. A one-dimensional particle code is used to corroborate the analytic theory. This code shows several interesting properties when the emitted current exceds the critical valuc. Chief among them is the presence of a turbulent microsheath near the cathode surface. The electrostatic potential in the gap's vacuum region is found to oscillate at a frequency that is quite insensitive to the emitted current and to the electrons' emission velocity. (C) 1994 American Institute of Physics.
\end{abstract}

When a sufficiently strong transverse magnetic field $B$ is imposed across an anode-cathode gap, electrons released from the cathode will not be able to reach the anode. Under the steady-state condition, that critical magnetic field is the Hull cutoff value $B_{H}=\left(2 m V / e D^{2}\right)^{1 / 2}$, where $D$ is the gap spacing, $V$ is the gap voltage, $e$ is the electron charge, and $m$ is the electron mass.' The electrons are assumed to be emitted with zero velocity. It is important to note that $B_{\mathrm{H}}$ is independent of the emitted current density, $J$.

In this Letter, we examine the regime $B>B_{\mathrm{H}}$. Specifically, we present an analytic expression that gives the critical emission current density, $J_{C}$, above which steady state solution ceases to exist. We employ a particle-in-cell (PIC) code ${ }^{2}$ to examine the dynamical behavior of the gap, both below and above this critical current.

It is anticipated, on physical grounds, that such a critical current exists. If the emission current is vanishingly small, electrons are expected to follow the familiar single particle orbits in the external electric and magnetic fields, which are uniform. A collection of such particles represents a timeindependent, laminar solution. If, on the other hand, electrons are released in great quantities, steady-state solutions are not expected to exist, as seen from the familiar experiences with the virtual cathode. ${ }^{3}$ The latter regime may only be treated via particle simulations. The transition from laminar to turbulent solutions in a crossed-field gap is the subject of this Letter.

This seemingly old problem is of considerable current interest, especially in the study of ultralow noise crossedfield amplifiers (CFA's). In many CFA's of practical significance, the clectrons are released from the cathode (which is part of the interaction region) by secondary emission, and the operating magnetic field is always higher than $B_{\mathrm{H}}$. How to control electron emission so as to reduce the amplifier noise has remained a very important consideration. ${ }^{4}$ It, in part, motivates the present study.

The above mentioned critical current is most easily established when the electrons are emitted normal to the cathode with zero velocity. Consider the emitted current being raised quasistatically, from a very low value. All electrons are initially accelerated by the vacuum electric field inside the gap. They all turn back before reaching the anode if $B>B_{\mathrm{H}}$. As the emitted current is raised, the initially accelerating electric field right in front of the cathode is reduced. For zero injection velocity, therefore, the steady-state solution fails to exist when the emitted current is sufficiently high to render the surface electric field equal to zero. Thus the critical current, for this $B>B_{\mathrm{H}}$ case, is simply determined from the space-charge-limited condition when the electrons are released with zero velocity.

Consider a time-independent, one-dimensional planar, nonrelativistic model. In the Cartesian coordinates, the cathode is located at $x=0$, and the anode at $x=D$, held at potential $V$ with respect to the cathode. The external magnetic field is $\hat{z} B$. Let $\phi(x)$ be the self-consistent electrostatic potential, and $u(x)$ be the (non-negative) velocity component of the cold electrons in the $x$ direction. Using conservation of energy, $e \phi(x)=\frac{1}{2} m\left(u^{2}+\Omega^{2} x^{2}\right)$, we may write the Poisson equation

$$
\frac{d^{2} \phi}{d x^{2}}=\frac{e n}{\epsilon_{0}}=\frac{2 J}{\epsilon_{0} u}
$$

in the Llewellyn form (see e.g., Birdsall and Bridges ${ }^{3}$ ):

$$
\frac{d^{2} u}{d t^{2}}+\Omega^{2} u=\frac{2 e J}{m \epsilon_{0}}
$$




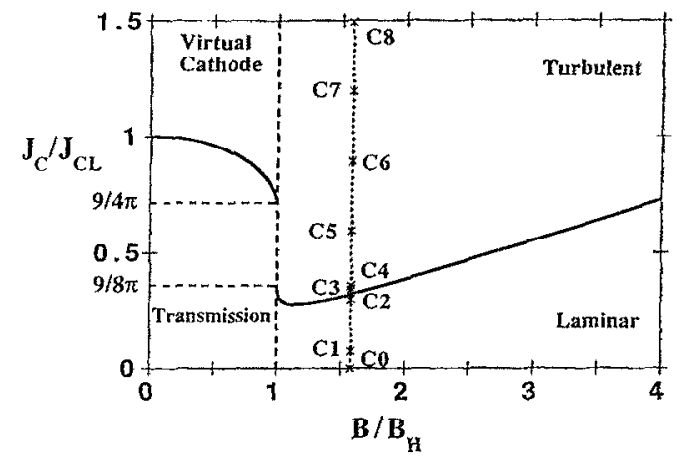

FIG. 1. The critical current density (in units of the Child -Langmuir value) above which steady state solutions cease to exist, as a function of the magnetic field $B$ (in units of the Hull cutoff value), for zero electron emission velocity. The points $\mathrm{C} 0, \mathrm{Cl}, \ldots, \mathrm{C} 8$ mark the cases simulated.

where $\Omega=e B / m$ is the cyclotron frequency and $t=\int d x / u$. In Eq. (1), we have used the continuity equation, $n u$ $=$ constant, as well as the fact that the electron density, $n$, is due to the departing electrons and the returning electrons, thus giving rise to a factor of 2 in the last term of Eqs. (1) and (2). The emitted current density, $J$, is a constant.

In terms of the nondimensional variables, $\tilde{x}=x / D, \tilde{t}$ $=\Omega t, \tilde{J}=e J / m \epsilon_{0} \Omega^{3} D, \tilde{\phi}=e \phi / m D^{2} \Omega^{2}, \tilde{V}=e V / m D^{2} \Omega^{2}$, $\tilde{u}=u / \Omega D$, the differential equation (2) may be integrated to yield $\tilde{u}(\tilde{t})=2 \tilde{J}[1-\cos (\tilde{t})]$, under the condition that the electron is initially at rest and nonaccelerated (space-chargelimited condition). This solution may be integrated again to yield $\tilde{x}(\tilde{t})=2 \tilde{J}[\tilde{t}-\sin (\tilde{t})]$. From these simple solutions, we see that an electron begins to be turned back when $\tilde{t}=2 \pi$, at which $\tilde{x}=\tilde{x}_{T}=4 \pi \tilde{J}$, and $\tilde{u}=0$. Note that $\tilde{x}_{T}$ represents the maximum excursion of electrons (i.e., "hub height") when the critical current is reached. It is easy to show that, at $\tilde{x}=\tilde{x}_{T}$, the normalized electric potential is $\tilde{\phi}_{T}=\tilde{x}_{T}^{2} / 2=8 \pi^{2} \tilde{J}^{2}$, and the normalized elcctric field is $\tilde{E}_{T}=-2 \tilde{J} \tilde{t}_{T}=-4 \pi \tilde{J}$.

The last statement allows us to express the anode voltage as the sum of $\tilde{\phi}_{T}$ and the potential drop in the vacuum region, $\tilde{x}_{T}<\tilde{x}<1: \tilde{V}=\tilde{\phi}_{T}-\tilde{E}_{T}\left(1-\tilde{x}_{T}\right)$. This in turns yields $\tilde{V}=4 \pi \tilde{J}-8 \pi^{2} \tilde{J}^{2}$. The last expression may be easily solved for $\tilde{J}$ in terms of $\tilde{V}$. This gives the critical current $\tilde{J}_{C}$ as a function of gap voltage $\tilde{V}$. When normalized to the ChildLangmuir limiting current, $\tilde{J}_{\mathrm{CL}}=(2 / 9)(2 \tilde{V})^{3 / 2}$, this critical current reads

$$
\frac{\tilde{J}_{C}}{\tilde{J}_{\mathrm{CL}}}=\left(\frac{9}{8 \pi}\right)\left(\frac{B}{B_{\mathrm{H}}}\right)^{3}\left[1-\sqrt{1-\left(\frac{B_{\mathrm{H}}}{B}\right)^{2}}\right],
$$

where we have used $\tilde{V}=\left(B_{\mathrm{H}} / B\right)^{2} / 2$.

The expression (3) is valid for $B>B_{\mathrm{H}}$. It is shown in Fig. 1, which, for completeness, also includes the regime $B<B_{\mathrm{H}}$. The latter regime was recently reexamined in considerable detail. ${ }^{5,6}$ Thus Fig. 1 provides the critical value of the emitted current, above which time-independent solutions cease to exist, for general values of magnetic field. The discontinuity in $J_{\mathrm{C}}$ at $B=B_{\mathrm{H}}$ reflects a different state of the solutions, a fact observed by Pollack, ${ }^{7}$ interpreted as the limiting current, ${ }^{5}$ and reaffirmed in particle simulations by Verboncoeur and Birdsall. ${ }^{6}$ For the case $B>B_{\mathrm{H}}$, it may be
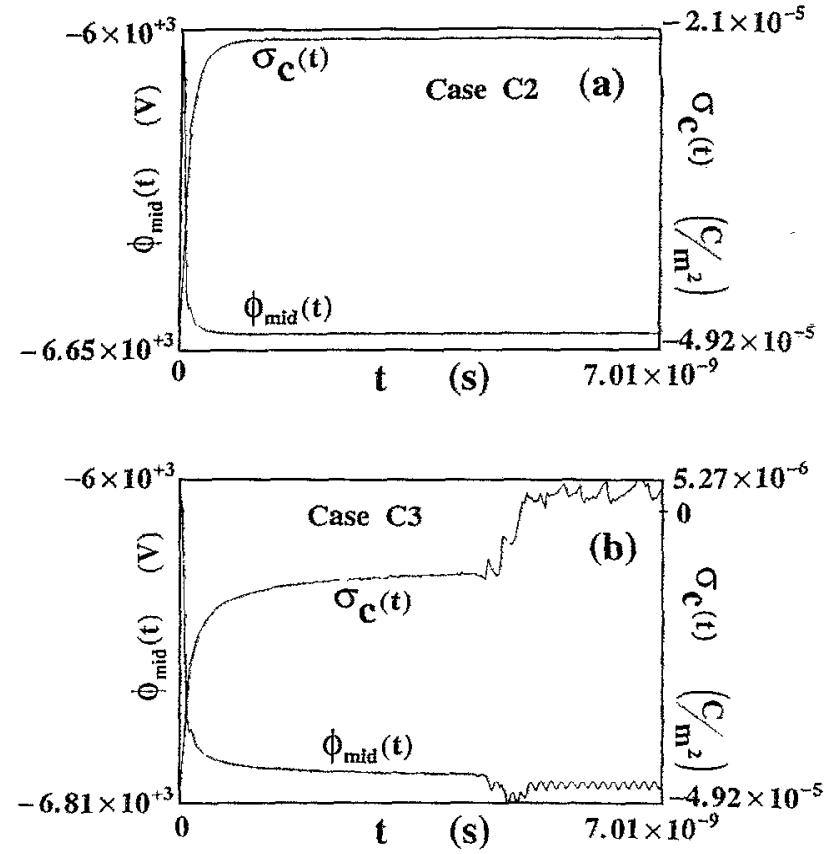

FIG. 2. The potential at the midpoint of the crossed-field gap, $\phi_{\text {mid }}$, and the cathode surface charge density, $\sigma_{c}$, as functions of time, for cases $\mathrm{C2}$ $\left(I / I_{c} \approx 0.95\right)$, and $\mathrm{C} 3\left(I / I_{c} \approx 1.10\right)$.

shown that as $J \rightarrow J_{C}$, the maximum electron excursion in $x$, $x_{T}$, is simply the Brillouin hub height, $D\left[1-\sqrt{1-\left(B_{\mathrm{H}} / B\right)^{2}}\right]$, which is always less than $D\left(B_{\mathrm{H}} / B\right)^{2}$, the latter being the maximum electron excursion when the space charge is negligible.

We have extensively studied the regime $B>B_{\mathrm{H}}$ using a PIC code, Plasma Device Planar 1 Dimensional ${ }^{2}$ (PDP1), as the injected current is increased (case $\mathrm{C} 0, \ldots \mathrm{C} 8$ in Fig. 1). In the simulations, Figs. 2-4, we fixed $D=0.00216 \mathrm{~m}, B=0.27$ T, cathode area $A=0.001492 \mathrm{~m}^{2}$, the cathode was held at $V_{c}=-12000 \mathrm{~V}$, while the anode was grounded. Thus $V=12000 \mathrm{~V}, B_{\mathrm{H}}=0.171 \mathrm{~T}, B / B_{\mathrm{H}}=1.579, J_{C}=2.1 \times 10^{5}$ $\mathrm{A} / \mathrm{m}^{2}$, and $I_{C}=A J_{C}=313 \mathrm{~A}$. The electrons were injected as a cold beam normal to the cathode with, unless otherwise stated, injection energy of $1 / 2 \mathrm{eV}$. [After several attempts, we
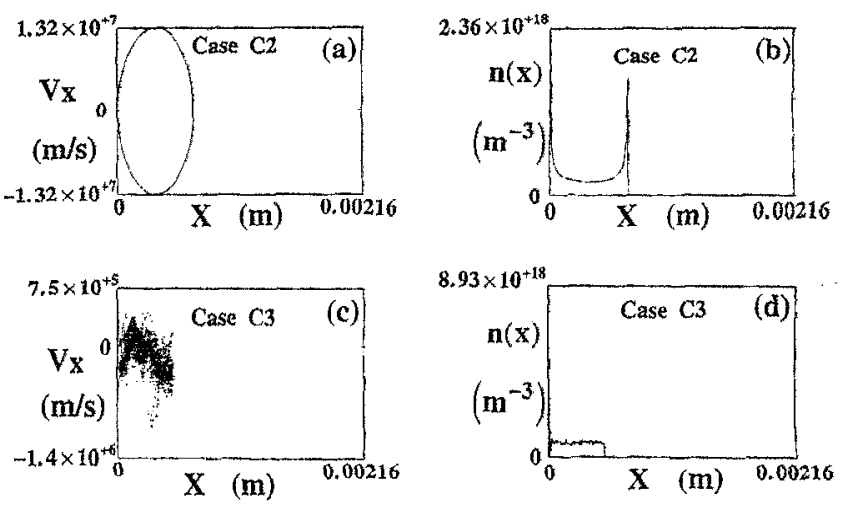

FIG. 3. The phase-space plot, and the electron density distribution, $n(x)$, for cases $\mathrm{C} 2\left(I / I_{c} \approx 0.95\right)$ and $\mathrm{C} 3\left(I / I_{c} \approx 1.10\right)$. 


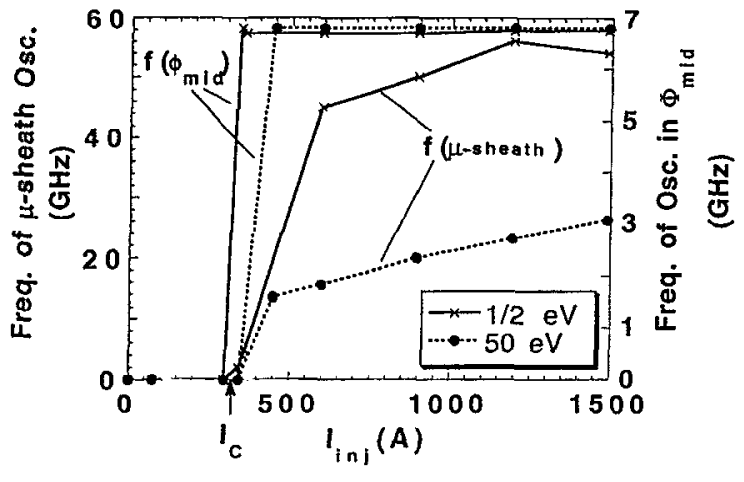

FIG. 4. The frequencies of oscillation of the surface charge density, $\sigma_{c}$, (i.e., of the microsheath on the cathode surface), and of the midpotential, $\phi_{\text {mid }}$, as functions of the injected current, $I=I_{\text {inj }}$. Two injection energies $(1 / 2$ and $50 \mathrm{eV})$ were simulated.

failed to generalize Eq. (3) to include such a nonzero injection energy. $\left.{ }^{8}\right]$

The transition from laminar to turbulent behavior is clearly shown from the runs below transition of case $\mathrm{C} 2$ $(I=A J=298 \mathrm{amps})$ and above transition, case C3 $(I=A J$ $=343 \mathrm{~A})$. For case $\mathrm{C} 2$, the potential at midway between the anode-cathode gap, $\phi_{\text {mid }}(t)$, and the cathode surface charge density, $\sigma_{c}(t)$, remain asymptotically constant [Fig. 2(a)], whereas those for case $\mathrm{C} 3$ break into oscillations for time $t \geqslant 4 \mathrm{~ns}$ [Fig. 2(b)]. The phase-space plot and density profile for case C2 [Figs. 3(a) and 3(b)] are dictated by single particle motion. The trajectory of one electron is repeated by all others, and this property is shared by low injection current cases, $\mathrm{C} 0$ and $\mathrm{Cl}$ in Fig. 1. The corresponding figure [Fig. 3(c)] for case C3 shows turbulent behavior: the phase space is randomized and the $x$ component of the particle velocity is substantially reduced. The space-charge density profile [Fig. 3(d)] is almost flattened, extending from the cathode to roughly the Brillouin hub height. This is true for all abovetransition cases $\mathrm{C} 3, \mathrm{C} 4, \ldots, \mathrm{C} 8$ (Fig. 1). The surface charge on the cathode [Fig. 2(b)] is positive, i.e., a potential minimum is always formed in front of the cathode, when turbulent behaviors emerge from the numerical simulation. The electric field on the cathode is positive, having a mean value on the order of $4 \mathrm{kV} / \mathrm{cm}$. This latter value is shared by all above-transition cases $\mathrm{C} 3, \mathrm{C} 4, \ldots, \mathrm{C} 8$. The depth of this potential minimum is on the order of the injection energy that is given to the electrons in the code. Thus, a microsheath is always formed right in front of the cathode for low injection velocity when the injected current exceeds the critical value (Fig. 1). This sheath oscillates at a frequency that varies according to the emitted current and to the emission velocity (Fig. 4). No electrons reach the anode in any of our runs for $B=1.597 B_{\mathrm{H}}$. In fact, no electrons reach the midpoint, $x=D /$ 2 , in the gap. ${ }^{9}$ The potential at this midpoint oscillates at a frequency that is quite independent of the injected current
(Fig. 4), of the initial energy of the electron (up to $200 \mathrm{eV}$ ), and of the grid size and number of computer particles used in the simulations. Neither the microsheath oscillation frequency, nor the oscillation frequency in the midpotential corresponds to the cyclotron frequency, the plasma frequency, nor to the upper hybrid frequency. This characteristic is shared by all cases $\mathrm{C} 4, \mathrm{C} 5, \ldots, \mathrm{C} 8$ in Fig. 1. The physical origin of the oscillations remains to be examined.

In summary, this study seems to have added substance to the notion that a necessary condition for quiescent behavior in a crossed-field gap is that electron emission be limited. The criterion is given by Eq. (3) for $B>B_{\mathrm{H}}$, and is confirmed by particle simulation.

\section{ACKNOWLEDGMENTS}

We are obliged to $\mathrm{C}$. K. Birdsall and J. P. Verboncoeur for their generous assistance and for their kind interest in this work. We would also like to acknowledge helpful discussions with D. Chernin, N. Dionne, R. M. Gilgenbach, T. Ruden, and J. Saloom.

This work was supported by the Naval Research Laboratory, and by the Office of Naval Research.

\footnotetext{
${ }^{1}$ A. W. Hull, Phys. Rev. 18, 31 (1921).
}

${ }^{2}$ PDPl (Plasma Device Planar 1 Dimensional), $11990-93$ Regents of the University of California, Plasma Theory and Simulation Group, Berkeley, California. Available from Software Distribution Office of the ILP, 205 Cory Hall, Berkeley, California 94720, software@eecs.Berkeley.EDU. This is a one-dimensional electrostatic code that includes all three components of velocity.

${ }^{3}$ See, e.g., C. K. Birdsall and W. B. Bridges, Electron Dynamics of Diode Regions (Academic, New York, 1966); R. C. Davidson, Physics of Nonneutral Plasmas (Addison-Wesley, Redwood City, CA, 1990); High Power Microwaves, edited by V. L. Granatstein and I. Alexeff (Artech House, Norwood, MA, 1987).

${ }^{4}$ D. Chernin, A. Drobot, and M. Kress, Digest of IEEE International Electron Devices Meeting (Institute of Electrical and Electronic Engineers, New York, 1993), IEEE Cat. No. 93CH3361-3, p. 773

${ }^{5}$ Y. Y. Lau, P. J. Christenson, and David Chernin, Phys. Fluids B 5, 4486 (1993), and references therein.

${ }^{\sigma} \mathrm{J} . \mathrm{P}$. Verboncoeur and C. K. Birdsall, in IEEE International Conference on Plasma Sciences, Santa Fe, New Mexico, June 1994 (Institute of Electrical and Electronic Engineers, New York, 1994), IEEE Cat. No. 94CH3465-2, p. 226.

${ }^{7}$ M. A. Pollack and J. R. Whinnery, IEEE Trans. Electron Devices ED-11, 81 (1964); T. Van Duzer, ibid. ED-8, 78 (1961); ED-10, 370 (1963).

${ }^{8}$ Secondary electrons are typically emitted with an initial energy of at least a few eV's, much higher than the cathode temperature. See, e.g., O. Hachenberg and W. Brauer, in Advances in Electronics and Electron Physics, edited by L. Martin (Academic, New York, 1959), p. 413; R. O. Jenkins and W. G. Trodden, Electron and Ion Emission from Solids (Dover, New York, 1965), p. 54; A. J. Dekker, in Solid State Physics, Advances in Research and Applications, edited by F. Seitz and D. Turnbull (Academic, New York, 1958), Vol. 6, p. 251. The importance of these secondary electrons to crossed-field amplifiers is addressed recently by $\mathrm{J}$. R. M. Vaugham, IEEE Trans. Electron Devices ED-36, 1963 (1989), and by A. Shih and C. Hor, ibid. ED-40, 824 (1993).

${ }^{9}$ Diocotron instabilities are ruled out in this paper since there is no variation in the fields in any direction parallel to the cathode surface. Thus our findings do not contradict the experiments by T. J. Orzechowski and G. Bekefi, Phys. Fluids 22, 978 (1979). 